\title{
Posterior cervical angle and cervical length in predicting the successful induction of labour in nulliparous woman
}

\author{
Original Karam Mohamed Bayoumy, Amr Helmy Yehia, Mohammed Mahmoud Salman, \\ Article Nada Magdy Alkady*
}

Department of Obstetrics and Gynecology, Faculty of Medicine, Ain-Shams University, Cairo, Egypt

\begin{abstract}
Objective:To assess the value of pre-induction sonographic assessment of the posterior cervical angle (PCA) and cervical length in the prediction of successful induction of labor (IOL).

Patients and Methods:The present prospective observational study included IOL candidates who had their PCA and cervical length assessed by transvaginal ultrasonography and the Bishop score at the Obstetrics and Gynecology Department, Ain-Shams Maternity Hospital, Cairo, Egypt, from $1^{\text {st }}$ November 2018 to $31^{\text {st }}$ May 2019. The accuracy of these tests in predicting successful IOL (defined as vaginal delivery) was compared.

Results: The analysis included 41 women with successful IOL and 29 women with unsuccessful IOL. The suggested cutoffs for the prediction of successful IOL were a PCA of more than $100^{\circ}$, a cervical length of less than $31 \mathrm{~mm}$, and a Bishop score of more than four. The areas under the receiver operating characteristics curves for these three measures were not significantly different. However, a PCA of more than $100^{\circ}$ had the best sensitivity (87.80\%), specificity (86.21), positive predictive value (90\%), negative predictive value $(83.3 \%)$ compared with the other two predictors.

Conclusion: Posterior cervical angle had the best accuracy in predicting successful induction of labor compared with the cervical length and the Bishop score.
\end{abstract}

Key Words: Cervical length, induction of labor, posterior cervical angle

Received: 3 October 2019, Accepted: 4 October 2019

Corresponding Author: Nada Magdy Hassan, Department of Obstetrics and Gynecology, Faculty of Medicine, Ain-Shams University, Cairo, Egypt, Tel.: 01118718712, E-mail: nadonado9@yahoo.com

ISSN: 2090-7625, February 2020, Vol.10, No.1

\section{INTRODUCTION}

Induction of labor nowadays is a common procedure in obstetric practice: it is used in $30-40 \%$ of women in labor $^{[1]}$. Failure of induction of labor is not uncommon it is approximately about $25 \%$ in nulliparous and $5 \%$ in multiparous cases Therefore, the accurate selection of candidates for successful induction of labor represents an important issue in obstetric practice ${ }^{[2]}$. Successful induction depends largely on cervical characteristics. The Bishop Score by digital cervical examination is the gold standard established method ${ }^{[3]}$. However, some investigators have raised concerns about the value of the Bishop score in predicting the induction of labor outcome because it is a subjective measure with great variability affected by a physician's clinical experience ${ }^{[4]}$. According to these limitations, it is important to find a better alternative to the established method. Transvaginal ultrasound appears promising because of its availability, reproducibility, and ability to provide images for accurate documentation and inter observer comparison ${ }^{[5]}$. Several ultrasonography parameters have been mentioned as substitutes to the traditional Bishop score. Especially investigators have looked at the role of the cervical length measured by transvaginal ultrasonography prior to induction of labor $^{[6]}$. Only a few studies have focused on the value of pre induction measurement of posterior cervical angle in prediction the induction of labor outcome ${ }^{[7]}$. Because results was conflicting. More studies are needed to evaluate the cervical characteristics before the induction of labor and to recognize reliable predictors for success of induction.

\section{AIM OF THE WORK}

The aim of the present study is to assess the value of pre-induction sonographic assessment of the posterior cervical angle and cervical length in the prediction of successful induction.

\section{PATIENTS AND METHODS}

In this study, sonographic cervical length and posterior cervical angle were investigated to predict the successful labor induction. The study was conducted at Ain-Shams 
University Maternity Hospital from $1^{\text {st }}$ November 2018 to $31^{\text {st }}$ May 2019 after Ethical approval from the Ethics Committee, Obs/Gyn Department, Faculty of Medicine, Ain-Shams University. Informed consent was obtained from the women before the procedure. This prospective study recruited 90 pregnant women attending Prelabor Unit at Ain-Shams University Maternity Hospital ; 20 women were primary excluded and 70 women were included in the study. The study included candidates for induction of labor aged 18-35 years who had a single live fetus in cephalic presentation with gestational age of 37-41 weeks and no history of uterine scar and absence of active labor.The exclusion criteria were fetal anomalies, placenta previa, prepartum hemorrhage, cephalopelvic disproportion, morbid obesity (body mass index more than 40) and fetal macrosomia (fetal weight of $4 \mathrm{~kg}$ or more).

All women in this study were subjected to the following ; full history taking including personal history, menstrual history, obstetric history, history of present pregnancy, medical and surgical history. General examination including vital sings, body mass index, abdominal examination to exclude scars of previous abdominal or pelvic surgeries and assess fundal level and fetal presentation. Then digital vaginal examination was done to all women to assess bishop score, membrane status, pelvic adequacy and ensure presentation. All women underwent ultrasonographic examination by skilled obstetrician using a Medison SonoAce R5 ultrasonography machine. Trans-abdominal ultrasonography was done first to check the fetal number, viability, biometry, presentation, adequacy of amniotic fluid and placental site. Then, the vaginal probe was gently inserted into the vagina. A sagittal plane of the cervix was obtained to ensure visualization of the entire length of the cervical canal. Once the view was optimal, the depth was increased so that the image was occupied approximately two-thirds of the screen. Then, cervical length was measured ; it is defined as a line between the internal and the external cervical orifices. After measuring cervical length, the posterior cervical angle was measured with the software for measuring angle in an ultrasonography. Picture was taken in the sagittal plane at the level of the internal cervical os and the angle was assessed at the junction of line measuring the cervical length and posterior uterine wall. Protocol of induction of labor was done using ASUMH protocol of induction. Misoprostol (vagiprost $25 \mathrm{mcg}$ ) was inserted in the posterior vaginal fornix and the cervix was reassessed after 4 hours. If no cervical ripening was achieved second dose of one tablet of misoprostol (vagiprost $25 \mathrm{mcg}$ ) was used and to be repeated after 4 hours if needed. If no cervical ripening achieved (no cervical dilatation or effacement were noted) after the five doses of misoprostol (at 4 hours intervals), the induction was considered to be failed induction of labor and the infant was delivered by cesarean delivery in accordance with the hospital's policy for induction of labor. Women with a soft cervix and those who progressed after misoprostol administration received an intravenous infusion of oxytocin (Syntocinon; Novartis, Basel, Switzerland). The starting dose was 5 units (in $500 \mathrm{~mL}$ Ringer Solution) at a rate 12 drops/minute ; the dose was doubled every 30 minute until efficient contractions were achieved. The partogram was done at the beginning of the induction process and the continuous cardiotocography was used in all cases. A successful outcome of IOL was defined as an uncomplicated vaginal delivery, whereas unsuccessful IOL was defined as cesarean delivery because of failed progress of labor. Women who had a cesarean delivery for reasons other than failed IOL, such as prepartum hemorrhage and fetal distress were excluded from the data analysis.

Data were fed to the computer and analyzed using IBM SPSS software package version 20.0. (Armonk, NY: IBM Corp) Qualitative data were described using number and percent. Quantitative data were described using range (minimum and maximum), mean, standard deviation and median. Significance of the obtained results was judged at the $5 \%$ level.

The used tests were: Chi-square test Fisher's Exact, Student t-test, Pearson coeffienet, ROC, sensitivity, specificity, PPV, NPV, and regression.

\section{RESULTS}

In total, 90 candidates for IOL were screened for eligibility (Figure 1). After exclusions, 70 women were included in the final data analysis; IOL was successful in $41(58.6 \%)$ women and unsuccessful in $29(41.4 \%)$.

The maternal age, body mass index, gestational age and neonatal weight did not differ significantly between the two groups (Table 1).

The indication for IOL was classified into two categories; passed due date $(\mathrm{n}=12)$ and other indications $(n=58)$ which included rupture of membranes, decrease fetal kicks and maternal medical disorders such as gestational diabetes and gestational hypertension.

There was no significant correlation found between cause of induction and the successful induction. The two groups differed significantly in each of three cervical measures ; the Bishop score was higher, the cervical length was shorter and the PCA was larger in the group with successful IOL.

Logistic regression analysis revealed that rupture of membranes, Bishop Score, cervical length and PCA were significantly correlated with the IOL outcome (Table 2).

In the multivariate regression analysis including these four factors, the only correlation found was between the IOL outcome and PCA. The best cut-off values for 
the prediction of successful IOL were a PCA of more than $100^{\circ}$, a cervical length of less than $31 \mathrm{~mm}$ and a Bishop score of more than 4 (Table 3).

There was a significant difference between the PCA, cervical length and Bishop Score as regards the overall accuracy, as evidenced by comparison of the AUC values (0.93 vs. 0.66 vs. 0.65$)$, respectively. A PCA of more than $100^{\circ}$ had the best sensitivity, specificity and positive predictive value, negative predictive value in comparison with the cervical length and the Bishop Score (Figure 2).

Table 1: Clinical and demographic date of study group

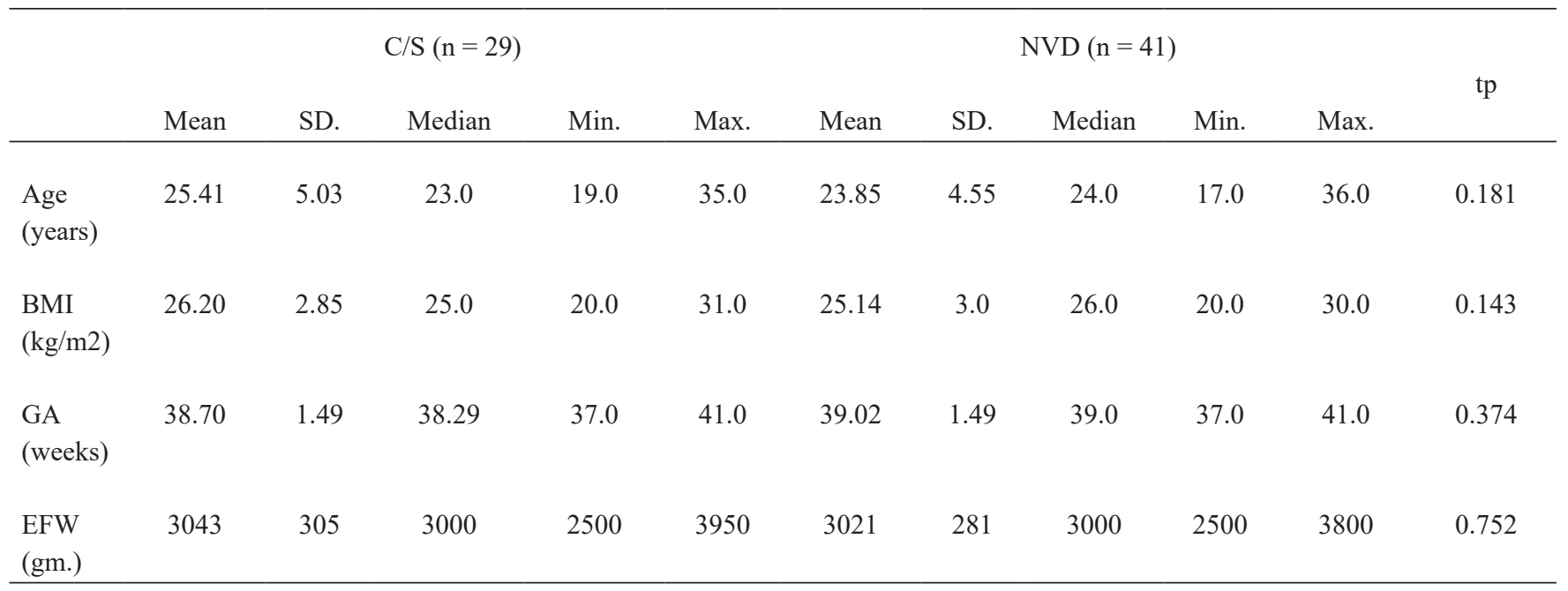

Table 2: Univariate and multivariate analysis for the parameters affecting successful induction of labor

\begin{tabular}{lcccc}
\hline \multirow{2}{*}{ NVD cases } & \multicolumn{2}{c}{ Univariate } & \multicolumn{2}{c}{ \#Multivariate } \\
\cline { 2 - 5 } & $P$ & OR $(95 \%$ C.I $)$ & $p$ & OR $(95 \%$ C.I $)$ \\
\hline Bishop score & $0.018^{*}$ & $2.632^{*}(1.179-5.878)$ & 0.265 & $2.027(0.585-7.020)$ \\
Intact membrane & $0.012^{*}$ & $0.270 *(0.097-0.752)$ & 0.137 & $0.293(0.058-1.479)$ \\
Cervical length & $0.022^{*}$ & $0.835^{*}(0.716-0.974)$ & 0.081 & $1.199(0.978-1.470)$ \\
PCA $(0)$ & $<0.001 *$ & $1.181 *(1.093-1.277)$ & $<0.001 *$ & $1.217 *(1.098-1.349)$ \\
\hline
\end{tabular}

$p$ value is 0.0006

Table 3: Agreement (sensitivity, specificity) for different parameters to predict successful outcome

\begin{tabular}{|c|c|c|c|c|c|c|c|c|}
\hline & AUC & $p$ & $95 \%$ C.I & Cut off & Sensitivity & Specificity & PPV & NPV \\
\hline Bishop score & $0.652 *$ & $0.031^{*}$ & $0.523-0.782$ & $>4$ & 34.15 & 86.21 & 77.8 & 48.1 \\
\hline $\begin{array}{l}\text { Cervical } \\
\text { length }\end{array}$ & $0.664 *$ & $0.020^{*}$ & $0.532-0.795$ & $\leq 31$ & 63.41 & 58.62 & 68.4 & 53.1 \\
\hline PCA & $0.931 *$ & $<0.001^{*}$ & $0.862-1.000$ & $>100$ & 87.80 & 86.21 & 90.0 & 83.3 \\
\hline
\end{tabular}

$p$ value is 0.0002 


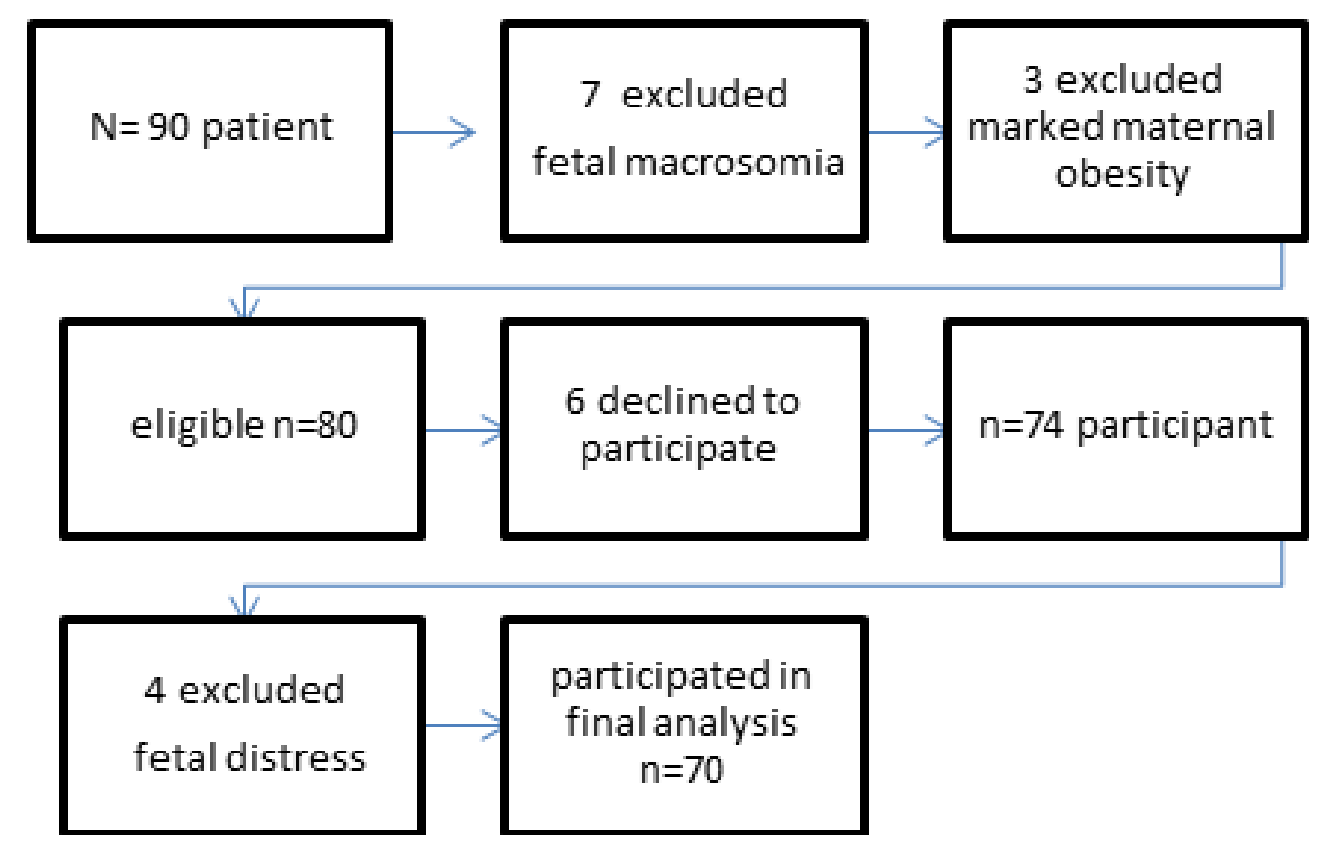

Fig. 1: Flow chart of population study

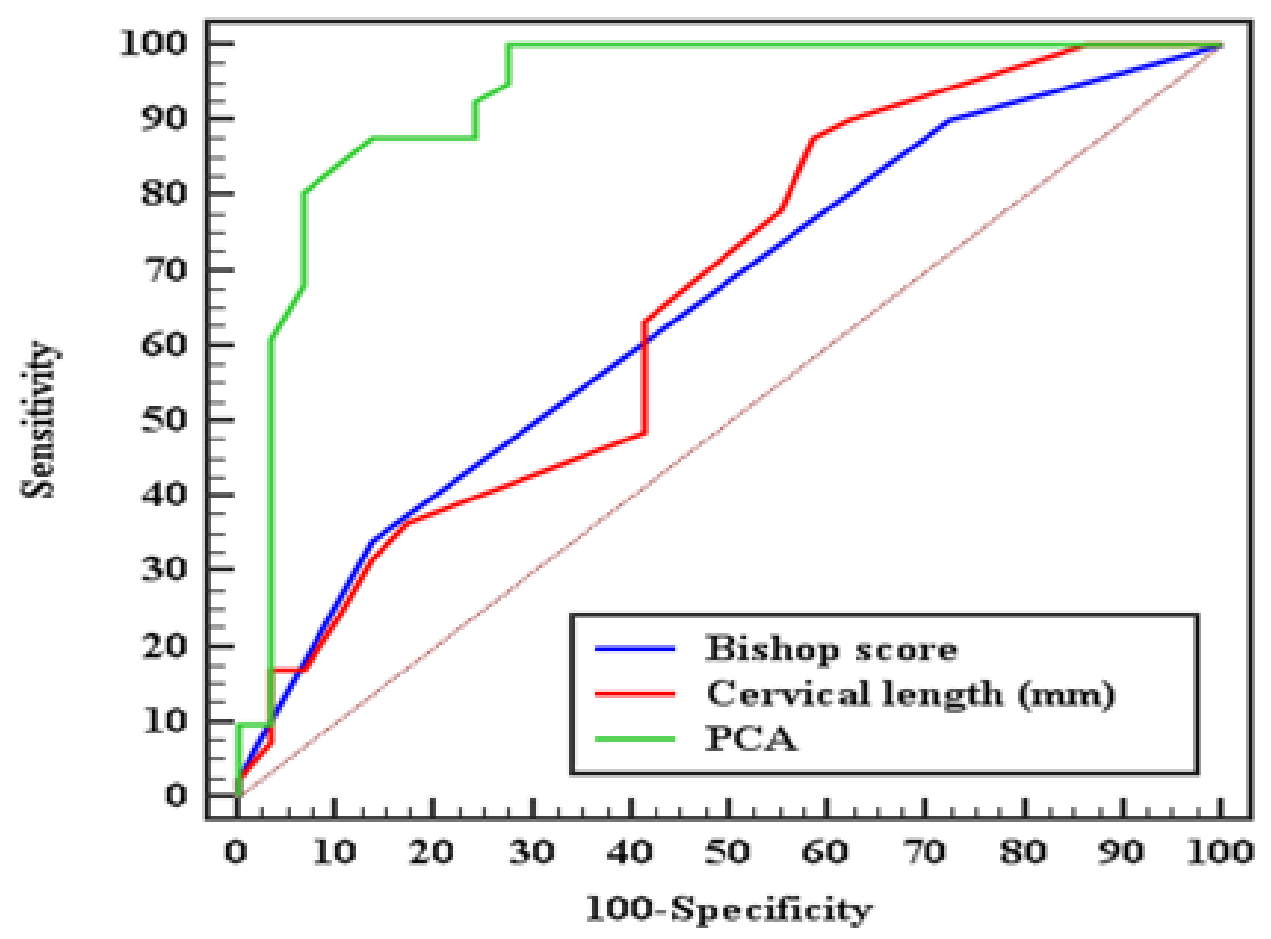

Fig 2: ROC curve for different parameters to predict successful outcome 


\section{DISCUSSION}

The present study assesses the accuracy of the PCA, cervical length and Bishop score in the prediction of successful IOL. Multivariate regression analysis proved the validity of PCA in predicting the outcome of IOL because it was an independent factor affecting the outcome of IOL. The PCA had a higher specificity, positive predictive value, and negative predictive value than did cervical length measurement and the Bishop score.

The results imply that the predictive power of the PCA is considerably greater than that of the other two cervical tests. Concern over the poor predictive value of the Bishop score has already been raised by previous authors. Papillon-Smith and Abenhaim ${ }^{[8]}$ in analysis of 32 observational studies they established the superiority of ultrasonography cervical length assessment over traditional digital examination in predicting the IOL outcome.

Few studies to date have evaluated the role of PCA measurement in predicting the outcome of IOL. Aladwy et al., 2018 who studied induction of labor in 70 women at the Obstetrics and Gynecology Department, Kasr El-Aini Hospital and found that PCA ,cervical length and bishop score were not significantly different but PCA more than 99.5 had the best accuracy in predicting successful IOL with sensitivity $(91.84 \%)$ and specificity $(90.4 \%)$.

Paterson-Brown et al. ${ }^{[9]}$ reported that the PCA was more accurate than the Bishop score in predicting a vaginal delivery. A PCA of more than $70^{\circ}$ together with a Bishop score of more than five had the best accuracy for predicting a successful IOL outcome. Rane et al. ${ }^{[10]}$ reported that a PCA of more than $120^{\circ}$ was associated with a positive response to IOL within 24 hours. The identification of different PCA cut-offs is most likely attributable to the large variability in methodology among these studies.

This current study was a prospective observational study that conducted at Ain-Shams University Maternity Hospital. Prior to induction of labor pregnant women underwent digital cervical assessment by Bishop Score performed by skilled obstetrician.

Then, trans-abdominal ultrasound was done followed by transvaginal ultrasound. Ultrasound examination was performed by different skilled obstetrician who was masked to the digital examination In this study, we included only nulliparous women to exclude the effect of parity on cervical response to IOL.
Another important aspect of this study was that only two methods were used as induction agents (misoprostol and oxytocin) to minimize the possible influence on the outcome. There was a wide variability of study protocols used in other studies; it is possible that parity and different induction agents have an impact on the duration as well as the mode of delivery.

This study had their limitations duo to digital examination to assess bishop score was done by different skilled obstetricians who may give different measures. In this study we didn't demonstrate the effect of fetal occipital position in predicting the outcome of induction of labor

\section{CONCLUSION}

Posterior cervical angle more than 100 had the best accuracy in predicting successful induction compared with cervical length and bishop score. So, we recommend the routine use of sonographic cervical measurements, in particular measurement of the PCA, in the assessment of IOL candidates to achieve a better outcome.

\section{CONFLICT OF INTEREST}

There are no conflicts of interests.

\section{REFERENCES}

1. Harper LM, Caughey AB, Odibo AO, Roehl KA, Zhao $\mathrm{Q}$ and Cahill AG. Normal progress of induced labor. Obstet Gynecol. 2012; 119:1113-1118.

2. Bahadori F, Ayatollahi H, Naghavi-Bhzad M, Khalkhali H, Naseri Z, et al. Methods for assessing pre-induction cervical ripening. Cochrane Database Syst Rev. 2015; (6): Cd010762.

3. Faltin-traub EF, Boulvain M, Faltin DL, Extermann $\mathrm{P}$ and Irion $\mathrm{O}$. Reliability of the Bishop Score before labour induction at term. Eur J Obstet Gynecol Reprod Biol. 2004; 112(2): 178-81.

4. Kolkman DG, Verhoeven CJ, Brinkhorst SJ, et al. The Bishop score as a predictor of labor induction success: A systematic review. Am J Perinatol. 2013; 30:625-630

5. Ezebialu IU, Eke AC, Eleje GU and Nwachukwu CE. Methods for assessing pre-induction cervical ripening. Cochrane Database Syst Rev. 2015; 6: Cd010762. 
6. Al-Adwy AM, Sobh SM, Belal DS, Omran EF, Hassan A, Saad AH, Afifi MM and Adel MN. Diagnostic accuracy of posterior cervical angle and cervical length in the prediction of successful induction of labor.2018

7. Gokturk U, Cavkaytar $\mathrm{S}$ and Danisman N. Can measurement of cervical length, fetal head position and posterior cervical angle be an alternative method to Bishop Score in the prediction of successful labor induction? J Matern Fetal Neonatal Med. 2014; 28:1360-1365.
8. Papillon-Smith J, Abenhaim HA. The role of sonographic cervical length in labor induction at term. J Clin Ultrasound. 2015; 43:7-16

9. Paterson-Brown S, Fisk NM, Edmonds DK, Rodeck $\mathrm{CH}$. Preinduction cervical assessment by Bishop's score and transvaginal ultrasound. Eur $\mathrm{J}$ Obstet Gynecol Reprod Biol. 1991; 40:17-23

10. Rane SM, Guirgis RR, Higgins B, Nicolaides $\mathrm{KH}$. The value of ultrasound in the prediction of successful induction of labor. Ultrasound Obstet Gynecol. 2004; 24:538-549. 